\title{
Effect of corticosteroids on post-intubation tracheal stenosis
}

\author{
JOSEPH BRAIDY, GUY BRETON, LOUISE CLEMMENT \\ From the Department of Medicine, Respiratory Division, and Department of Radiology, Hôpital Saint-Luc, \\ University of Montreal, Montreal, Canada
}

\begin{abstract}
A 57 year old patient presented with progressive tracheal stenosis two months after intubation. An intraluminal polypoid lesion was found at the site of the cuff of the endotracheal tube. It disappeared within five days of treatment with inhaled beclomethasone. Further improvement of forced expiratory and inspiratory flow occurred after systemic corticosteroid treatment, with resolution of peritracheal oedema. Topical and systemic corticosteroids may be useful in the management of early post-intubation tracheal stenosis.
\end{abstract}

Tracheal stenosis, a rare complication of intubation, is most likely to occur in a critically ill patient requiring prolonged intubation.' Substantial stenosis must be present for symptoms to occur. Once established and causing symptoms, tracheal stenosis is generally treated surgically or by endoscopic dilatation. We report a case where severe tracheal stenosis occurred after a relatively short period of intubation and responded favourably to topical and systemic corticosteroid treatment.

\section{Case report}

A 57 year old woman was admitted to the hospital for dyspnoea. Two months previously she had undergone a nephrostomy for urinary tract infection and a left ureteral calculus. Surgery was complicated by Escherichia coli septicaemia and ventricular fibrillation. She was initially intubated for $\mathbf{4 8}$ hours but had to be reintubated for another five days because of acute renal failure and the adult respiratory distress syndrome. On extubation bronchoscopy showed severe laryngeal oedema with granulation tissue over the posterior aspect of the larynx and at the site of the endotracheal cuff. Intubation was required for four more days. A No 8 endotracheal tube with a low pressure cuff was used on each occasion (Sheridan Corporation, Argyle, New York). The patient recovered slowly and was discharged. Over the subsequent month she noticed progressively increasing dyspnoea of such a degree that she became breathless while dressing.

Address for reprint requests: Dr J Braidy, Pneumology, Hôpital SaintLuc, 1058 rue Saint-Denis, Montréal, Quebec H2X 3J4, Canada.

Accepted 4 May 1989
On admission (two months after intubation) she was in moderate respiratory distress, with a breathing rate of $24 / \mathrm{min}$, and was using her accessory inspiratory muscles. Her breathing was noisy and became louder with hyperventilation. The voice was normal. A left nephrostomy tube was draining clear urine.

Fibreoptic bronchoscopy showed normal vocal cords. The trachea was narrowed to a $6 \mathrm{~mm}$ opening in the middle third. A $2 \mathrm{~mm}$ polypoid mass was present at that level on the anterior aspect of the trachea. The mucosa was diffusely hyperaemic above this level. The tracheobronchial tree could not be explored beyond the narrowed area. A biopsy was not performed because of the severity of the obstruction. Computed tomography of the trachea (figs $1 a$ and $1 b$ ) showed extensive peritracheal oedema. The surface at the site of maximal stenosis was $15 \%$ of the normal tracheal surface on several sections. Her flow-volume curve (fig 2) showed features of severe "fixed" tracheal obstruction with a reduction in peak expiratory flow to $2 \mathrm{l} / \mathrm{s}$ (predicted $7 \mathrm{l} / \mathrm{s}$ ), a "plateau" of the expiratory curve of about $1 \mathrm{l} / \mathrm{s}$, and a similar reduction of the maximal inspiratory flow.

Beclomethasone aerosol treatment was started (200 $\mu \mathrm{g}$ every six hours). The patient felt better within a few days. A flow-volume curve done five days after the beginning of treatment showed improvement in the expiratory and to a lesser degree the inspiratory flow at all lung volumes. Fibreoptic bronchoscopy, performed the same day with the purpose of burning the granulation tissue with a laser, showed that, although the oedema persisted, the polypoid mass had disappeared.

Over the subsequent week no further improvement occurred. Prednisone was started orally at $1 \mathrm{mg} / \mathrm{kg}$ in a single daily morning dose. Two weeks later the flow-volume loop showed substantial improvement, though there was still a persistent expiratory plateau. The patient was discharged on a decreasing regimen of prednisone. After six weeks of treatment the flow-volume curve had improved further (fig 2). Computed tomography showed that the peritracheal oedema had resolved and the narrowed area was now about half of the normal tracheal section.

\section{Discussion}

Tracheal stenosis occurs in about $20 \%$ of critically ill patients requiring prolonged intubation; it develops in the subglottic area or at the level of the cuff and does not usually result in more than $50 \%$ narrowing of the tracheal lumen.' Our patient had a more severe stenosis (about an $85 \%$ reduction in the surface area), even though a low pressure cuff was used. Her initial stormy course with ventricular fibrillation and repeated intubation could have contributed to this outcome. 


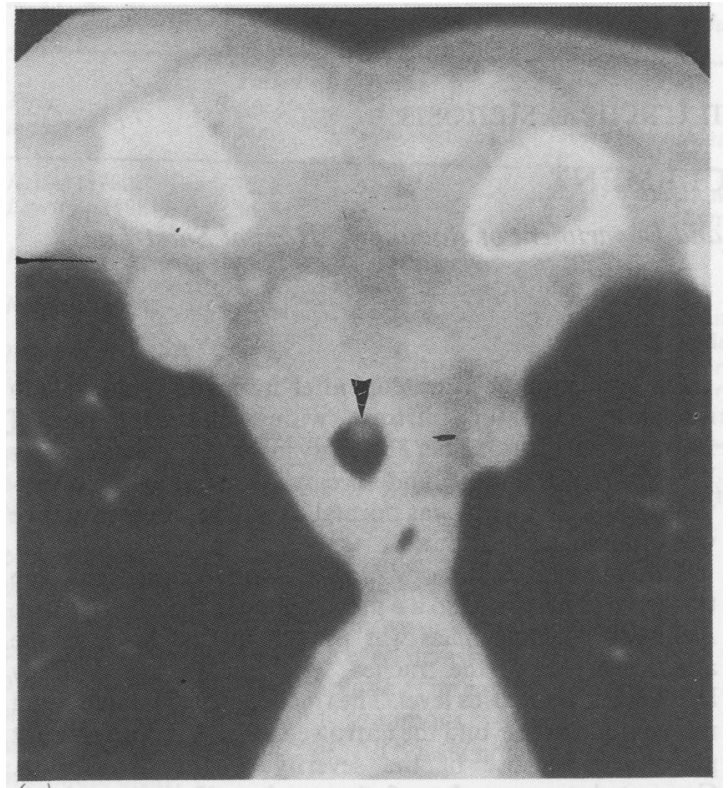

(a)

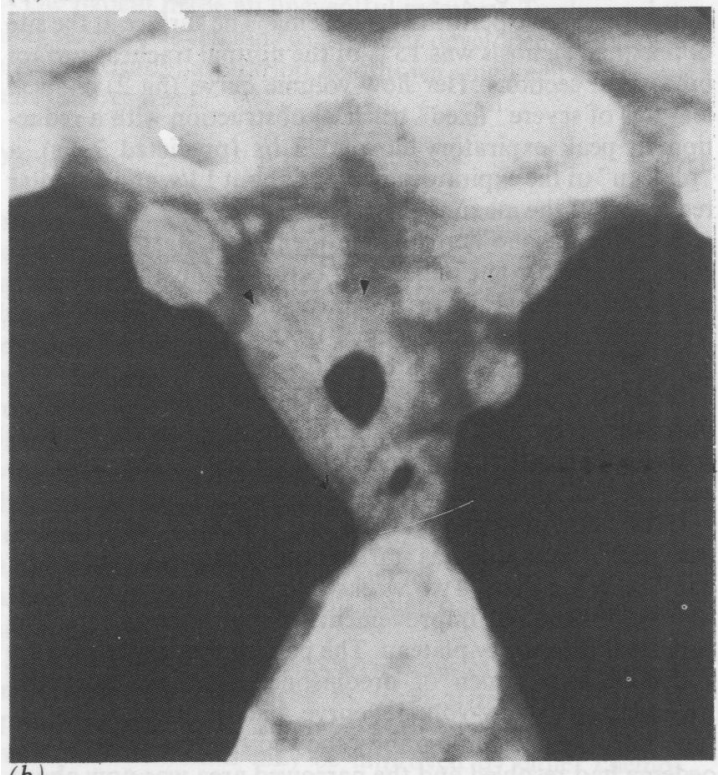

(b)

Fig 1 Computed tomogram of the trachea at the level of maximal stenosis, (a) with large window setting (large arrowhead indicates intraluminal polypoid lesion) and (b) with a narrower window setting (small arrowheads indicate peritracheal oedema)

While the patient is breathing through a rigid tube substantial reduction of flow does not occur unless the internal diameter of the tube reaches $10 \mathrm{~mm} \cdot{ }^{23}$ Further narrowing results in a dramatic fall in peak expiratory flow (PEF); at $6 \mathrm{~mm}$ the PEF is about $20 \%$ of control values. Because of this particular relation between flow and airway

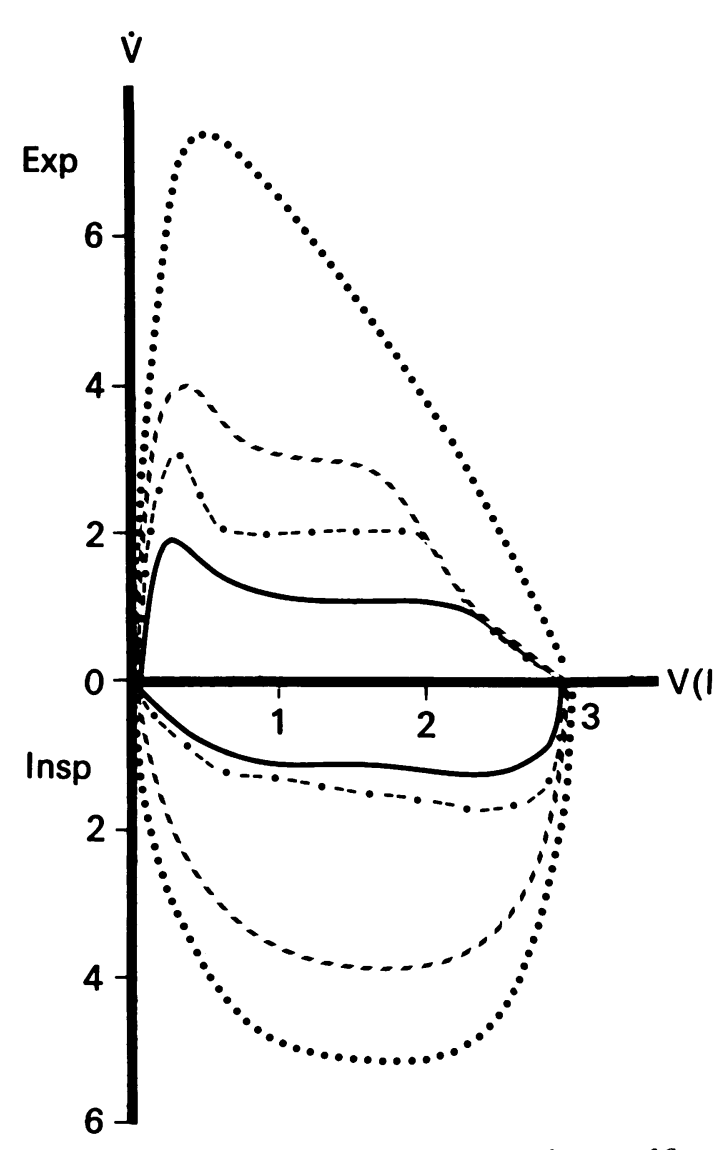

Fig 2 Flow-volume curves, showing severe reduction of flow $(\dot{V})$ at all lung volumes ( $V$ ) during both inspiration (insp) and expiration (exp) with a "plateau," typical of "fixed" large airway obstruction. - On admission; $\bullet-\cdots$ five days after beclomethasone; _. . . six weeks after prednisone; ..... predicted values.

diameter, a small improvement in a severely narrowed airway윽 may result in substantial relief of airflow limitation. This $>$ presumably explains the improvement in our patient's dys-음 pnoea when the small polypoid lesion in her trachea resolved
while substantial stenosis was still present.

Once established, tracheal stenosis is usually treated $N$ surgically. ${ }^{4}$ Corticosteroids combined with antibiotics have been shown to improve the outcome of subglottic tracheal stenosis in a dog model. ${ }^{5}$ Intraluminal stenting with intralesional and systemic corticosteroids has been used inco children with subglottic stenosis but no long term follow up $\bar{\varnothing}$ was reported. ${ }^{6}$ In our patient the rapid initial response to ${ }^{+}$ topical beclomethasone with resolution of polypoid granula- $\square$ tion tissue could be explained by the subacute nature of the $\overline{+}$ inflammation occurring at the site of the endotracheal cuff. $\mathbb{D}$ The subsequent resolution of the peritracheal oedema with $\frac{}{\mathbb{D}}$ systemic corticosteroid treatment was associated with further improvement of air flow and of dyspnoea. 
We believe that a trial of corticosteroids is indicated when post-intubation tracheal stenosis is diagnosed early. It may influence the inflammatory response favourably and preclude the need for reconstructive surgery. We cannot rule out the possibility that our patient would have improved without treatment; but the inflammatory nature of the lesion (as suggested by endoscopy and radiology), the progressive deterioration in symptoms over one month, and the rapid response to corticosteroids (five days) suggest a therapeutic response to steroids. Serial flow-volume loop measurements and computed tomography are helpful in assessing the physiological and anatomical extent of the stenosis.

We wish to thank Drs J Martin and P Renzi for editorial help and thoughtful comments and Miss $\mathrm{Z}$ Hammoud for secretarial assistance.

\section{References}

1 Stauffer J, Olson D, Petty T. Complications and consequences of endotracheal intubation and tracheostomy. A prospective study of 150 critically ill adult patients. Am J Med 1981;70:65-76.

2 Miller RD, Hyatt RE. Obstructing lesions of the larynx and trachea: clinical and physiologic characteristics. Mayo Clin Proc 1969;44:145-68.

3 Al-Bazzaz F, Grillo H, Kazemi H. Response to exercise in upper airway obstruction. Am Rev Respir Dis 1975;111:631-40.

4 Grillo H. Primary reconstruction of airway after resection of subglottic laryngeal and upper tracheal stenosis. Ann Thorac Surg 1982;33:3-18.

5 Croft C, Boronwiecki B. Therapy of iatrogenic subglottic stenosis: a steroid/antibiotic regimen. Laryngoscope 1979;89:482-9.

6 Othersen B. The technique of intraluminal stenting and steroid administration in the treatment of tracheal stenosis in children. J Pediatr Surg 1974;9:683-90. 